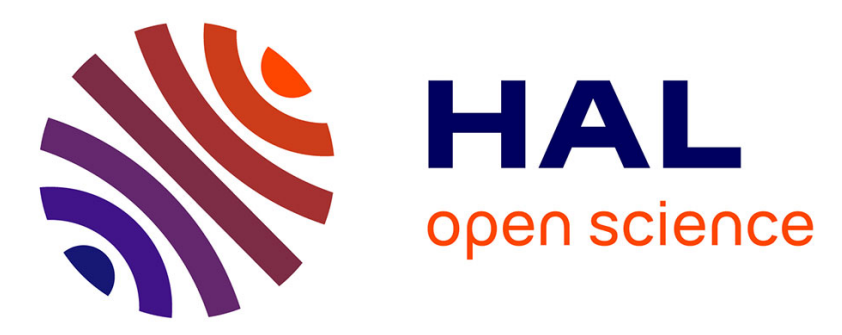

\title{
On the asymptotic analysis of Littlewood's reliability model for modular software
}

James Ledoux

\section{To cite this version:}

James Ledoux. On the asymptotic analysis of Littlewood's reliability model for modular software. B. H. Lindqvist, K. A. Doksum. Mathematical and Statistical Methods in Reliability, World Scientific, pp.521-536, 2003. hal-00916262

\section{HAL Id: hal-00916262 \\ https://hal.science/hal-00916262}

Submitted on 9 Dec 2013

HAL is a multi-disciplinary open access archive for the deposit and dissemination of scientific research documents, whether they are published or not. The documents may come from teaching and research institutions in France or abroad, or from public or private research centers.
L'archive ouverte pluridisciplinaire HAL, est destinée au dépôt et à la diffusion de documents scientifiques de niveau recherche, publiés ou non, émanant des établissements d'enseignement et de recherche français ou étrangers, des laboratoires publics ou privés. 


\title{
ON THE ASYMPTOTIC ANALYSIS OF LITTLEWOOD'S RELIABILITY MODEL FOR MODULAR SOFTWARE
}

\author{
James Ledoux \\ Centre de Mathmatiques INSA $\&$ IRMAR \\ INSA, 20 avenue des Buttes de Cosmes, \\ 35043 Rennes Cedex, \\ France \\ E-mail: james.ledoux@insa-rennes.fr
}

\begin{abstract}
We consider a Markovian model, proposed by Littlewood, to assess the reliability of a modular software. Specifically, we are interested in the asymptotic properties of the corresponding failure point process. We focus on its time-stationary version and on its behavior when reliability growth takes place. We prove the convergence in distribution of the failure point process to a Poisson process. Additionally, we provide a convergence rate using the distance in variation. This is heavily based on a similar result of Kabanov, Liptser and Shiryayev, for a doubly-stochastic Poisson process where the intensity is governed by a Markov process.
\end{abstract}

\section{Introduction}

Two approaches are used in the stochastic modeling of the failure process of a software. The prevalent approach adopts the so-called black-box view of the system, where only the interactions with the environment are considered. The martingale methods ${ }^{3}$ for analyzing point processes, provide an unified framework for almost all published black-box models. To each model corresponds a specific stochastic intensity of the point process. ${ }^{22,10}$ A second approach, called the white-box approach, incorporates information on the structure of the software in models. Littlewood's model ${ }^{14}$ is the most popular model resulting from this approach. It has inspired most other works. Littlewood proposed a Markov-type model for the reliability assessment of modular software. For a software with a finite number of modules: 
- the structure of the software is represented by an irreducible finite continuous time Markov chain $X=\left(X_{t}\right)_{t \geq 0}$, where $X_{t}$ is the active module at time $t$.

- When module $i$ is active, failure times are part of a homogeneous Poisson Process (HPP) with intensity $\mu(i)$.

- When control switches from module $i$ to module $j$, a failure may happen with probability $\mu(i, j)$.

- When a failure appears, the execution is assumed to be resumed instantaneously. So, a failure does not affect the software.

- All failure processes are assumed to be independent, given a sequence of activated modules.

This could be considered to be a naive model. For instance, at any time $t$, the control of the execution is assumed to be in only one module. However, using a suitable definition of the state space of the Markov process $X$, the model may be adapted to a software system where the control is shared by several of its parts. In the Littlewood model, the failures do not affect the execution, the control structure is Markovian, ... A large number of theses assumptions are questionable, but some of them may be addressed. We refer the reader to ${ }^{11}$ for a discussion on these issues and to ${ }^{7}$ for a recent survey on the architecture-based software reliability.

An important issue in reliability theory, specifically for software systems, is what happens when the failure parameters tend to be smaller and smaller. Littlewood claimed: ${ }^{14}$

As all failure parameters $\mu(i), \mu(i, j)$ tend to zero, the failure process described above is asymptotically an HPP with intensity

$$
\lambda=\sum_{i} \pi(i)\left(\sum_{j} Q(i, j) \mu(i, j)+\mu(i)\right)
$$

where $Q=(Q(i, j)), \pi$ are the generator and the stationary distribution of $X$, respectively. This statement is well-known in the community of software reliability and has widely supported the hierarchical approach for assessing the dependability of modular softwares. ${ }^{7}$ However, to the best of our knowledge, no proof of this fact is reported in the applied probability literature.

The purpose of this chapter is to provide precise statements on the asymptotic properties of the failure point process associated with the Littlewood model. Firstly, we discuss its time-stationary version. Intuitively, this corresponds to place the origin of the observation of the process at an "arbitrary time" $t$ (with large $t$ ). Secondly, we focus on the main contri- 
butions of this chapter, which are : first, a rigorous proof to the convergence results announced by Littlewood ${ }^{14,15}$; second, a rate of convergence. The derivation of our results will be based on the martingale approach for analyzing point processes.

The chapter is organized as follows. Section 2 gives the mathematical specification of the Littlewood model. Section 3 addresses the timestationary version of the failure point process. Section 4 is devoted to its Poisson approximation. First, we give the compensator and the stochastic intensity of the point process. Then, we prove the convergence of the finitedimensional distributions of the counting process to those of a homogeneous Poisson process with intensity $\lambda$ in (1). In the final part, a convergence rate for the convergence of these finite-dimensional distributions is provided.

\section{Mathematical Formulation of the Failure Point Process}

Let us consider the successive times at which a failure occurs $T_{n}, n \geq 1$, and assume that $T_{0}=0$. We denote the number of failures in the interval ] $0, t]$ by $N_{t}$

$$
N_{t}= \begin{cases}\max \left\{n \geq 1: T_{n} \leq t\right\} & \text { if } T_{1} \leq t \\ 0 & \text { if } T_{1}>t .\end{cases}
$$

We denote the finite set of modules $\{1, \ldots, M\}$ by $\mathcal{M} . X$ will be called the environment or execution process. Considering the bivariate process $Z=\left(N_{t}, X_{t}\right)_{t \geq 0}$ is very appealing from a mathematical and computational point of view. Indeed, it is easily seen from the assumptions on Littlewood's model, that $Z$ is a jump Markov process over the state space $S=\mathbb{N} \times \mathcal{M}$. The infinitesimal generator of this Markov process is

$$
G=\left(\begin{array}{cccc}
D_{0} & D_{1} & 0 & \cdots \\
0 & D_{0} & D_{1} & \ddots \\
\vdots & \ddots & \ddots & \ddots
\end{array}\right)
$$

when the states in $S$ are listed in lexicographic order. Matrices $D_{0}$ and $D_{1}$ are defined by

$$
\begin{aligned}
& \text { if } i \neq j: D_{0}(i, j)=Q(i, j)(1-\mu(i, j)) \quad D_{1}(i, j)=Q(i, j) \mu(i, j) \text {, } \\
& D_{0}(i, i)=-\sum_{j \neq i} Q(i, j)-\mu(i) \quad D_{1}(i, i)=\mu(i) .
\end{aligned}
$$

Note that $Q=D_{0}+D_{1}$ and $\lambda=\pi D_{1} \mathbb{I}^{\mathrm{t}}$ (see (1)), where $\mathbb{I}^{\mathrm{t}}$ denotes the $M$ dimensional column vector having all components equal to 1 . We point out that we deal with a failure point process that is a Markovian Arrival Process 
(MAP) as defined by Neuts. ${ }^{19,17}$ The Littlewood model has the (doublystochastic) Poisson process (driven) modulated by a Markov process as a special instance (setting parameters $\mu(\cdot, \cdot)$ to 0 ). We refer the reader to ${ }^{12}$ for the computational issue of various reliability metrics using the bivariate process $Z$.

As the observation duration $t$ tends to be large, we have the following asymptotic formula for the mean number of failures: ${ }^{12}$

$$
E\left[N_{t}\right]=\lambda t+(\alpha-\pi)\left(\mathbb{1}^{\mathrm{t}} \pi-Q\right)^{-1} D_{1} \mathbb{1}^{\mathrm{t}}+o(1)
$$

where $\alpha$ is the probability distribution of the random variable $X_{0}$ and $\lambda$ is defined in (1). We have as a result

$$
\lim _{t \rightarrow+\infty} \frac{E\left[N_{t}\right]}{t}=\lambda=\pi D_{1} \mathbb{I}^{\mathrm{t}}
$$

Thus, the mean value of the random variable $N_{t}$ has a linear asymptote when $t$ growths to infinity. As it can be expected from the last formula, the first moment of the counting process is $\lambda t$ when the environment process $X$ is stationary (that is, the probability distribution of $X_{n}$ 's is $\pi$ ). In such a case, we are concerned with the time-stationary version of the point process. This the core of the next section.

\section{Time-Stationary Version of the Failure Point Process}

In this section, we discuss the time-stationary version of the failure point process. That is, we give conditions under which the counting processes $\left(N_{t+s}-N_{t}\right)_{s \geq 0}$ (the point process viewed from time $t$ onwards) have the same distribution for all $t$. It is known that we get the time-stationary version of a MAP when its environment process $X$ is stationary. ${ }^{19,2}$ In fact, the question of time-stationarity for an MAP is related to the asymptotic properties of an associated Markov Renewal Process (MRP). Specifically, we need the stationary version of this MRP, which is stochastically equivalent to the MRP corresponding to an MAP with a stationary environment process $X$. This last property is well known (e.g. see ${ }^{19}$ ) but the details of the derivation are never reported. We give here, the main steps of the derivation to emphasize the interest in Markov modeling to get an analytic model that is tractable. Intensity $\lambda$ given in (1), also appears in the moments of the stationary version of the point process.

The MRP associated with Littlewood's failure model is defined as follows. ${ }^{17}$ Let us consider the marked point process $\left(T_{n}, J_{n}\right)_{n \geq 0}$ where $J_{n}$ is the active module just after the $n$th failure time $T_{n}$. It is easily seen from the 
assumptions on Littlewood's model, that $\left(T_{n}, J_{n}\right)_{n \geq 0}$ is a recurrent finite MRP with semi-Markov kernel $\left(Q_{t}\right)_{t \geq 0}$ on $\mathcal{M}$

$$
\begin{aligned}
Q_{t}=\left(Q_{t}(i, j)\right)_{i, j \in \mathcal{M}} & =\int_{0}^{t} \exp \left(D_{0} s\right) d s D_{1} \\
& =\left(I-\exp \left(D_{0} t\right)\right)\left(-D_{0}\right)^{-1} D_{1} .
\end{aligned}
$$

That is, we have $\mathbb{P}\left\{J_{n+1}=j, T_{n+1}-T_{n} \leq t \mid J_{n}=i\right\}=Q_{t}(i, j)$ for any $n \geq 0$. We refer the reader to ${ }^{13}$ for the basic properties of Markov renewal processes. The Markov chain $\left(J_{n}\right)_{n \geq 0}$, with the state space $\mathcal{M}$, has the following transition probability matrix

$$
Q_{\infty}=\left(Q_{\infty}(i, j)\right)_{i, j \in \mathcal{M}}=\int_{0}^{\infty} \exp \left(D_{0} s\right) d s D_{1}=\left(-D_{0}\right)^{-1} D_{1} .
$$

We note that

$$
Q_{t}=Q_{\infty}-\exp \left(D_{0} t\right) Q_{\infty}
$$

The distribution function of the sojourn time in state $i \in \mathcal{M}$ is given by

$$
H_{t}(i)=\sum_{j \in \mathcal{M}} Q_{t}(i, j)=1-\left(\exp \left(D_{0} t\right) \mathbb{I}^{\mathrm{t}}\right)(i)
$$

Then, the mean sojourn time in state $i$ is

$$
m(i)=-\left(D_{0}{ }^{-1} \mathbb{I}^{\mathrm{t}}\right)(i) .
$$

We associate with the semi-kernel $\left(Q_{t}\right)_{t \geq 0}$, its semi-Markov process $\left(J_{N_{t}}\right)_{t \geq 0}$ :

$$
t \geq 0, \quad J_{N_{t}}=J_{n} \quad \text { if } T_{n} \leq t<T_{n+1} .
$$

We need the forward recurrence time process $\left(V_{t}\right)_{t \geq 0}$ defined by

$$
V_{t}=T_{N_{t}+1}-t
$$

(i.e. time until the next failure after $t$ ) and the process $\left(J_{N_{t}+1}\right)_{t \geq 0}$ (the active module just after the next failure after time $t$ ).

Now, we are interested in the stationary version of the $\operatorname{MRP}\left(T_{n}, J_{n}\right)_{n>0}$. In the definition of an MRP, the origin of the observation of the process is assumed to be an epoch of failure $\left(T_{0}=0\right)$. Intuitively, considering the stationary version corresponds to start the observation at an arbitrary time $t$ (with large $t$ ). Thus, the MRP is assumed to be in progress for a long time at the beginning of its observation. Formally, we get from the initial $\operatorname{MRP}\left(T_{n}, J_{n}\right)_{n>0}$, a delayed MRP $\left(\widetilde{T}_{n}, \widetilde{J}_{n}\right)_{n>0}$, for which the probability distribution of $\left(\widetilde{J}_{N_{t}}, \widetilde{J}_{N_{t}+1}, \widetilde{V}_{t}\right)$ (and of $\left(\widetilde{J}_{N_{t}}, \widetilde{V}_{t}\right)$ ) does not depend on time 
$t .^{20,4}$ The first transition of the new MRP is governed by the semi-Markov matrix

$$
\widetilde{Q}_{t}(i, j)=\frac{1}{m(i)} \int_{0}^{t}\left(Q_{\infty}(i, j)-Q_{s}(i, j)\right) d s .
$$

The others jumps are governed by the initial semi-Markov kernel $\left(Q_{t}\right)_{t \geq 0}$ (see (2)). The probability distribution $\widetilde{\pi}$ of $\widetilde{J}_{0}$ is

$$
\forall i \in \mathcal{M}, \quad \widetilde{\pi}(i)=\lim _{t \rightarrow \infty} \mathbb{P}\left\{J_{N_{t}}=i\right\}=\frac{\nu(i) m(i)}{\nu m^{\mathrm{t}}} .
$$

where $\nu$ is the stationary distribution of the Markov chain $\left(J_{n}\right)_{n \geq 0}$. It is easily checked by a direct computation that

$$
\nu=\frac{-\pi D_{0}}{-\pi D_{0} \mathbb{I}^{\mathrm{t}}}=\frac{\pi D_{1}}{\pi D_{1} \mathbb{I}^{\mathrm{t}}}
$$

with the stationary distribution $\pi$ of $X\left(\pi\left(D_{0}+D_{1}\right)=\mathbf{0}\right)$. Note that ${ }^{4}$

$$
\begin{aligned}
\widetilde{\pi}(i) \widetilde{Q}_{t}(i, j) & =\lim _{x \rightarrow \infty} \mathbb{P}\left\{J_{N_{x}}=i, J_{N_{x}+1}=j, V_{x} \leq t\right\} \\
\widetilde{\pi} \widetilde{Q}_{t} & =\left(\lim _{x \rightarrow \infty} \mathbb{P}\left\{J_{N_{x}+1}=j, V_{x} \leq t\right\}\right)_{j \in \mathcal{M}}
\end{aligned}
$$

We check that the stationary $\operatorname{MRP}\left(\widetilde{T}_{n}, \widetilde{J}_{n}\right)_{n \geq 0}$ is stochastically equivalent to the $\operatorname{MRP}\left(T_{n}, J_{n}\right)_{n \geq 0}$ with $\pi$ as initial distribution for the Markovian environment process $X$. Let us identify the (unconditional) probability distribution of $\left(\widetilde{T}_{1}, \widetilde{J}_{1}\right)$ :

$$
\begin{aligned}
\widetilde{\pi} \widetilde{Q}_{t} & =\frac{1}{\nu m^{\mathrm{t}}} \int_{0}^{t}\left(\nu Q_{\infty}-\nu Q_{s}\right) d s \quad \text { from }(5) \\
& =\frac{1}{\nu m^{\mathrm{t}}} \int_{0}^{t} \frac{-\pi D_{0}}{-\pi D_{0} \mathbb{I}^{\mathrm{t}}} \exp \left(D_{0} s\right)\left(-D_{0}\right)^{-1} D_{1} d s \quad \text { from (2), (3), (6) } \\
& =\frac{1}{\nu m^{\mathrm{t}}} \frac{1}{\pi D_{1} \mathbb{1}^{\mathrm{t}}} \int_{0}^{t} \pi \exp \left(D_{0} s\right) D_{1} d s .
\end{aligned}
$$

Now, it follows from (4) and (6) that $\nu m^{\mathrm{t}}=1 / \pi D_{1} \mathbb{I}^{\mathrm{t}}$. As a result of the previous statements, we have

$$
\widetilde{\pi} \widetilde{Q}_{t}=\pi Q_{t} .
$$

Therefore, if the probability distribution of $X_{0}$ is $\pi$, then $\left(T_{1}, J_{1}\right)$ and $\left(\widetilde{T}_{1}, \widetilde{J}_{1}\right)$ have the same distribution. Since the next failure times are governed by the same semi-Markov kernel $\left(Q_{t}\right)_{t \geq 0}$, we have the stochastic equivalence between $\left(\widetilde{T}_{n}, \widetilde{J}_{n}\right)_{n \geq 0}$ and the $\operatorname{MRP}\left(T_{n}, J_{n}\right)_{n \geq 0}$ with stationary Markovian environment process $X$. 
We provide now, some results concerning the moments associated with the stationary Littlewood model. The details of the derivation may be found in ${ }^{2,19}$ and are not reported here ( $\mathrm{see}^{18}$ for the non-stationary case). We assume until the end of this section that the execution process $X$ is stationary. The mean value function of the counting process $N$ is given by

$$
E_{\pi}\left[N_{t}\right]=\lambda t \quad \forall t \geq 0 .
$$

We see that the mean function $E_{\pi}\left[N_{t}\right]$ is identical to that of a homogeneous Poisson process with intensity $\lambda$. The scalar $\lambda$ may be interpreted as the mean number of failures per time unit for the stationary failure model. The variance function $\sigma_{\pi}^{2}(t)$ for our counting process is

$$
\begin{aligned}
\sigma_{\pi}^{2}(t)= & {\left[\lambda+2 \pi D_{0}\left(\mathbb{I}^{\mathrm{t}} \pi-Q\right)^{-1} D_{1} \mathbb{I}^{\mathrm{t}}-2 \lambda^{2}\right] t } \\
& -2 \pi D_{1}\left(I-\mathbb{I}^{\mathrm{t}} \pi\right)\left(\mathbb{I}^{\mathrm{t}} \pi-Q\right)^{-2} D_{1} \mathbb{I}^{\mathrm{t}} \\
& -2 \pi D_{1}\left(\mathbb{I}^{\mathrm{t}} \pi-\exp (Q t)\right)\left(\mathbb{I}^{\mathrm{t}} \pi-Q\right)^{-2} D_{1} \mathbb{I}^{\mathrm{t}} \quad \forall t \geq 0 .
\end{aligned}
$$

The first two terms give the linear asymptote of the variance function when $t$ growths to infinity (the last term converges to zero). Note that the variance $\sigma_{\pi}^{2}(t)$ cannot be identified to that of a Poisson process with intensity $\lambda$.

The last formula concerns the covariance function $C_{\pi}$ of the stationary failure point process. If $C_{\pi}\left(t, t^{\prime} ; t_{0}\right) \stackrel{\text { def }}{=} E_{\pi}\left[N_{t}\left(N_{t^{\prime}+t+t_{0}}-N_{t+t_{0}}\right)\right]-\lambda^{2} t t^{\prime}$ with $t, t^{\prime}>0$ and $t_{0} \geq 0$, then

$C_{\pi}\left(t, t^{\prime} ; t_{0}\right)=\pi D_{1}(I-\exp (Q t)) \exp \left(Q t_{0}\right)\left(I-\exp \left(Q t^{\prime}\right)\right)\left(\mathbb{I}^{\mathrm{t}} \pi-Q\right)^{-2} D_{1} \mathbb{I}^{\mathrm{t}}$. When $t_{0}$ tends to infinity, this covariance is negligible, i.e. $C_{\pi}\left(t, t^{\prime} ; t_{0}\right)=$ $o(1)$. The number of observed failures in the two disjoint intervals $] 0, t]$ and ]$\left.t+t_{0}, t+t_{0}+t^{\prime}\right]$ are asymptotically non-correlated with $t_{0}$.

\section{Asymptotic Property in case of Reliability Growth}

We turn to the main contribution of this chapter. For software systems, it can be expected that a phenomenon of reliability growth takes place with the corrective actions. Thus, we are interested in the behavior of the failure point process when the failure parameters become small. According to Littlewood, the point process tends to be an HPP with intensity $\lambda$ given in (1). A basic way to represent reliability growth is to introduce the perturbated failure parameters

$$
\varepsilon \mu(i), \varepsilon \mu(i, j), \quad i, j \in \mathcal{M}
$$

where $\varepsilon>0$ is a small parameter. Then, we investigate the convergence of the failure point process as $\varepsilon$ tends to 0 . 
First of all, we note that the Poisson approximation provided by Littlewood cannot hold as it is stated in ${ }^{14}$. Indeed, consider, as in ${ }^{16}$, the time to the first failure $T_{1}$ for the model with the failure parameters in (7). Its probability distribution cannot be asymptotically exponential with parameter $\lambda$ as $\epsilon \rightarrow 0$. With the simple model of a Poisson process modulated by a two-states Markov process, specified by

$$
Q=\left(\begin{array}{cc}
-1 & 1 \\
1 & -1
\end{array}\right) D_{1}^{(\varepsilon)}=\left(\begin{array}{cc}
\mu(1) \varepsilon & 0 \\
0 & \mu(2) \varepsilon
\end{array}\right) D_{0}^{(\varepsilon)}=Q-D_{1}^{(\varepsilon)} \quad(\mu(1) \neq \mu(2))
$$

and a stationary environment $X\left(\mathcal{L}\left(X_{0}\right)=\pi=(1 / 2,1 / 2)\right)$, we have

$$
\lim _{\varepsilon \rightarrow 0} \mathbb{P}\left\{T_{1}>t\right\}=1 \text {. }
$$

This agrees with intuition. But, if we investigate the asymptotic distribution of $T_{1}$ at time scale $t / \varepsilon$, then it is easily checked that

$$
\lim _{\varepsilon \rightarrow 0} \mathbb{P}\left\{T_{1}>t / \varepsilon\right\}=\exp \left(-\pi D_{1} \mathbb{I}^{\mathrm{t}} t\right) .
$$

Therefore, this suggests to deal with the counting process $N^{(\varepsilon)}=\left(N_{t}^{(\varepsilon)}\right)_{t \geq 0}$ defined by

$$
t \geq 0 \quad N_{t}^{(\varepsilon)}=N_{t / \varepsilon}
$$

where $N_{t}$ denotes, until the end of the section, the number of failures in the interval $] 0, t]$ for Littlewood's model with system (7) of failure parameters.

We heavily use the so-called martingale approach to analyze the counting process $N^{(\varepsilon)}$. The corresponding basic results used in this chapter are reported in Bremaud's book. ${ }^{3}$

\subsection{Compensator and Stochastic Intensity of the Counting Process N}

Considering the bivariate process $Z$ to analyze the counting process $N$, allows one to deal with a Markov process with discrete state space. One more time, we can take advantage of the powerful analytic theory for such a class of processes. $\mathcal{F}^{Z}=\left(\mathcal{F}_{t}^{Z}\right)_{t \geq 0}$ denotes the internal history of the bivariate Markov process $Z=(N, X)$. Due to the special structure of generator $G$ of $Z, N$ may be interpreted as the following counter of specific transitions in $Z$

$$
N_{t}=\sum_{(x, y) \in T} N_{t}(x, y) \quad \text { where } T=\{((n, i) ;(n+1, j)), \quad i, j \in \mathcal{M}, n \geq 0\}
$$


and $N_{t}(x, y)$ is the number of transitions from state $x$ to state $y$ at time $t$. It is well known ${ }^{3}$ that, for the counting process $N(x, y)=\left(N_{t}(x, y)\right)_{t \geq 0}$,

$$
N_{t}(x, y)-\int_{0}^{t} \mathbf{1}_{\left\{Z_{s-}=x\right\}} G(x, y) d s
$$

is a $\mathcal{F}^{Z}$-martingale. We denote the left limit at $t$ of $Z$ by $Z_{t-}$. The random functions

$$
\lambda_{t}(x, y)=\mathbf{1}_{\left\{Z_{t-}=x\right\}} G(x, y), \quad A_{t}(x, y)=\int_{0}^{t} \lambda_{s}(x, y) d s
$$

are the $\mathcal{F}^{Z}$-intensity and the $\mathcal{F}^{Z}$-compensator $\left(\mathcal{F}^{Z}\right.$-dual predictableprojection) of $N(x, y)$, respectively. Then it is easily seen that $\left(N_{t}-A_{t}\right)_{t \geq 0}$ where

$$
A_{t}=\sum_{(x, y) \in T} A_{t}(x, y)
$$

is a $\mathcal{F}^{Z}$-martingale. The $\mathcal{F}^{Z}$-intensity of $N$ is

$$
\begin{aligned}
\lambda_{t} & =\sum_{(x, y) \in T} \mathbf{1}_{\left\{Z_{t-}=x\right\}} G(x, y)=\sum_{j \in \mathcal{M}} D_{1}\left(X_{t-}, j\right) \\
& =\varepsilon\left(\mu\left(X_{t-}\right)+\sum_{j \neq X_{t-}} Q\left(X_{t-}, j\right) \mu\left(X_{t-}, j\right)\right) .
\end{aligned}
$$

\subsection{Convergence to a Poisson Process}

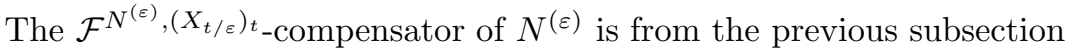

$$
A_{t}^{(\varepsilon)}=\sum_{i \in \mathcal{M}}\left(\mu(i)+\sum_{j \neq i} Q(i, j) \mu(i, j)\right) \varepsilon \int_{0}^{t / \varepsilon} \mathbf{1}_{\left\{X_{s-}=i\right\}} d s
$$

with intensity $\lambda_{t}^{(\varepsilon)}=\mu\left(X_{t / \varepsilon_{-}}\right)+\sum_{j \neq X_{t / \varepsilon_{-}}} Q\left(X_{t / \varepsilon_{-}}, j\right) \mu\left(X_{t / \varepsilon_{-}}, j\right)$. We are now in position to state the convergence of the Littlewood point process to a Poisson process as the small parameter $\varepsilon$ tends to zero.

Theorem 1: The probability vector $\pi$ is such that $\pi Q=0$. As $\varepsilon$ tends to 0 , the counting process $N^{(\varepsilon)}$ converges in distribution to the counting process $\left(P_{t}\right)_{t \geq 0}$ of an HPP with an intensity $\lambda$ defined by

$$
\lambda=\sum_{i} \pi(i)\left(\sum_{j \neq i} Q(i, j) \mu(i, j)+\mu(i)\right) .
$$


Proof: From the well known time-average properties of the cumulative process $\int_{0}^{t} f\left(X_{s}\right) d s$ for an irreducible finite Markov process $X$, we have

$$
\lim _{\varepsilon \rightarrow 0} \frac{\varepsilon}{t} \int_{0}^{t / \varepsilon} \mathbf{1}_{\left\{X_{s}=i\right\}} d s=\pi(i) \text { a.s. }
$$

where $\pi$ satisfies $\pi Q=0$. Thus, we derive from (8)

$$
\lim _{\varepsilon \rightarrow 0} A_{t}^{(\varepsilon)}=t \sum_{i \in \mathcal{M}} \pi(i)\left(\mu(i)+\sum_{j \neq i} Q(i, j) \mu(i, j)\right)=\lambda t \quad \text { a.s. . }
$$

In particular, this implies the convergence in probability of $A_{t}^{(\varepsilon)}$ to the compensator of an HPP with an intensity $\lambda$ as in (1). The theorem follows from Th $1^{8}$.

Remark 2: Littlewood extended his model $^{15}$ to consider sojourn times in each module with a general probability distribution. Thus, $X$ was assumed to be an irreducible semi-Markov process. He also identified ${ }^{15}$ the asymptotic distribution of its counting process to that of an HPP with the intensity

$$
\lambda^{*}=\frac{1}{\nu m^{\mathrm{t}}} \sum_{i \in \mathcal{M}} \nu(i)\left(\mu(i) \sum_{j} Q_{\infty}(i, j) m(i, j)+\sum_{j \neq i} Q_{\infty}(i, j) \mu(i, j)\right)
$$

where $Q_{\infty}$ is the transition probability matrix of the jump Markov chain associated with the semi-Markov process $X, \nu$ is such that $\nu Q_{\infty}=Q_{\infty}$ and $m(i, j)$ is the mean sojourn time in state $i$, given that the next state entered is $j$. We emphasize that a compensator approach leads to the above limiting Poisson process. Indeed, using the concept of compensator for marked

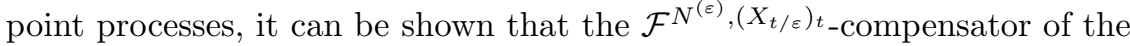
counting process $N^{(\varepsilon)}$ is given by

$$
\varepsilon\left[\int_{0}^{t / \varepsilon} \mu\left(X_{s-}\right) d s+\sum_{i} \sum_{j \neq i} \mu(i, j) A_{t / \varepsilon}(i, j)\right]
$$

where $A_{t}(i, j)$ is the compensator of the counting process $N_{t}(i, j)$ of transitions from state $i$ to state $j$ for $X$. For the asymptotic behavior of the first term, we just need time-average properties of cumulative process $\int_{0}^{t} f\left(X_{s}\right) d s$ for an irreducible finite semi-Markov process $X$, which are similar to those for the Markovian case. ${ }^{13}$ Using strong laws of large numbers for the second term, we get the convergence in distribution of $N^{(\varepsilon)}$ to an HPP with the intensity $\lambda^{*}$. Note that Anisimov and Korolyuk ${ }^{1}$ dealt with the case of a Poisson process with an intensity governed by an irreducible 
semi-Markov process $X$. This is the particular case where the $\mathcal{F}^{N^{(\varepsilon)},\left(X_{t / \varepsilon}\right)_{t_{-}}}$ compensator of $N^{(\varepsilon)}$ is given by

$$
\varepsilon \int_{0}^{t / \varepsilon} \mu\left(X_{s-}\right) d s
$$

(parameters $\mu(\cdot, \cdot)$ are assumed to be 0 ).

Note that $\lambda$ in (1) is the scalar product $\left\langle\pi, D_{1} \mathbb{I}^{\mathrm{t}}\right\rangle$. Moreover, if $Y_{s}^{(\varepsilon)}$ is vector $\left(\mathbf{1}_{\left\{X_{s / \varepsilon}=i\right\}}\right)_{i \in \mathcal{M}}$, then the compensator of $N^{(\varepsilon)}$ is from (8)

$$
A_{t}^{(\varepsilon)}=\int_{0}^{t}\left\langle Y_{s-}^{(\varepsilon)}, D_{1} \mathbb{I}^{\mathrm{t}}\right\rangle d s
$$

\subsection{Convergence Rate with Distance in Variation}

Let $T$ be any positive scalar. $\mathbb{T}$ is a finite subdivision $\left\{t_{0}, t_{1}, \ldots, t_{n}\right\}$ of the interval $[0, T]\left(0=t_{0}<t_{1}<\cdots<t_{n}=T\right)$. To evaluate the proximity between the respective probability distributions $\mathcal{L}\left(N_{\mathbb{T}}^{(\varepsilon)}\right)$ and $\mathcal{L}\left(P_{\mathbb{T}}\right)$ of $N_{\mathbb{T}}^{(\varepsilon)}=\left(N_{t_{1}}^{(\varepsilon)}, \ldots, N_{t_{n}}^{(\varepsilon)}\right)$ and $P_{\mathbb{T}}=\left(P_{t_{1}}, \ldots, P_{t_{n}}\right)$, we use their distance in total variation, denoted by $\mathrm{d}_{\mathrm{TV}}\left(\mathcal{L}\left(N_{\mathbb{T}}^{(\varepsilon)}\right), \mathcal{L}\left(P_{\mathbb{T}}\right)\right)$ :

$$
\mathrm{d}_{\mathrm{TV}}\left(\mathcal{L}\left(N_{\mathbb{T}}^{(\varepsilon)}\right), \mathcal{L}\left(P_{\mathbb{T}}\right)\right) \stackrel{\text { def }}{=} \sup _{B \subset \mathbb{N}^{n}}\left|\mathbb{P}\left\{N_{\mathbb{T}}^{(\varepsilon)} \in B\right\}-\mathbb{P}\left\{P_{\mathbb{T}} \in B\right\}\right| .
$$

For a locally bounded variation function $f(\cdot)$, the total variation in the interval $[0, T]$ is

$$
\operatorname{Var}_{[0, T]}(f) \stackrel{\text { def }}{=} \sup _{\mathbb{T} \in \mathcal{P}([0, T])} \sum_{i=1}^{n}\left|f\left(t_{i}\right)-f\left(t_{i-1}\right)\right|
$$

where $\mathcal{P}([0, T])$ is the set of all finite subdivisions of the interval $[0, T]$.

The main result of this subsection is based on the following estimate Th $3.1^{9}$

$$
\mathrm{d}_{\mathrm{TV}}\left(\mathcal{L}\left(N_{\mathbb{T}}^{(\varepsilon)}\right), \mathcal{L}\left(P_{\mathbb{T}}\right)\right) \leq E \operatorname{Var}_{[0, T]}\left(\widehat{A}^{(\varepsilon)}-A\right)
$$

where $\widehat{A}^{(\varepsilon)}, A$ are the $\mathcal{F}^{N^{(\varepsilon)}}$-compensator of $N^{(\varepsilon)}$ and the compensator of the HPP in Theorem 1 , respectively. $\widehat{A}^{(\varepsilon)}$ is from $(9)^{3}$

$$
\widehat{A}_{t}^{(\varepsilon)}=\int_{0}^{t}\left\langle\widehat{Y}_{s-}^{(\varepsilon)}, D_{1} \mathbb{I}^{\mathrm{t}}\right\rangle d s
$$


with $\widehat{Y}_{t}^{(\varepsilon)}=\left(\mathbb{P}\left\{X_{t / \varepsilon}=i \mid \mathcal{F}_{t}^{N^{(\varepsilon)}}\right\}\right)_{i \in \mathcal{M}}$. Therefore, we have from (10)

$$
\begin{aligned}
\mathrm{d}_{\mathrm{TV}}\left(\mathcal{L}\left(N_{\mathbb{T}}^{(\varepsilon)}\right), \mathcal{L}\left(P_{\mathbb{T}}\right)\right) & \leq E \operatorname{Var}_{[0, T]}\left(\int_{0}^{t}\left\langle\widehat{Y}_{s-}^{(\varepsilon)}-\pi, D_{1} \mathbb{I}^{\mathrm{t}}\right\rangle d s\right) \\
& \leq E \int_{0}^{T}\left|\left\langle\widehat{Y}_{s-}^{(\varepsilon)}-\pi, D_{1} \mathbb{I}^{\mathrm{t}}\right\rangle\right| d s .
\end{aligned}
$$

Note that $\left|\left\langle\widehat{Y}_{s}^{(\varepsilon)}-\pi, D_{1} \mathbb{I}^{\mathrm{t}}\right\rangle\right| \leq \delta\left\|\widehat{Y}_{s}^{(\varepsilon)}-\pi\right\|_{1}$ with $\delta=\max _{i \in \mathcal{M}}\left(\left(D_{1} \mathbb{I}^{\mathrm{t}}\right)(i)\right)-$ $\min _{i \in \mathcal{M}}\left(\left(D_{1} \mathbb{I}^{\mathrm{t}}\right)(i)\right)$ and $\|\cdot\|_{1}$ denotes the $l_{1}$-norm. Thus, it remains to estimate the convergence rate of $\left\|\widehat{Y}_{s}^{(\varepsilon)}-\pi\right\|_{1}$ to 0 when $\varepsilon \rightarrow 0$. The first step consists in writing a filtering equation for vector $Y_{t}^{(\varepsilon)}\left(\mathrm{Ch} \mathrm{IV}^{3}\right)$.

Lemma 3: Put $\widehat{Y}_{t}^{(\varepsilon)}=E\left[Y_{t}^{(\varepsilon)} \mid \mathcal{F}_{t}^{N^{(\varepsilon)}}\right]$. We have for all $t \geq 0$,

$$
\widehat{Y}_{t}^{(\varepsilon)}=\alpha+\frac{1}{\varepsilon} \int_{0}^{t} \widehat{Y}_{s-}^{(\varepsilon)} Q d s+\int_{0}^{t} v_{s-}^{(\varepsilon)}\left(d N_{s}^{(\varepsilon)}-\widehat{\lambda}_{s} d s\right)
$$

where $v_{s-}^{(\varepsilon)}=-\widehat{Y}_{s-}^{(\varepsilon)}+\widehat{Y}_{s-}^{(\varepsilon)} D_{1} / \widehat{\lambda}_{s}, \widehat{\lambda}_{s}=\left\langle\widehat{Y}_{s-}^{(\varepsilon)}, D_{1} \mathbb{I}^{\dagger}\right\rangle$ is the $\mathcal{F}^{N^{(\varepsilon)}}$-intensity of $N^{(\varepsilon)}$ and $\alpha$ is the probability distribution of $X_{0}$.

Proof: The MP $X^{(\varepsilon)}=\left(X_{t / \varepsilon}\right)_{t \geq 0}$ has the generator $Q^{(\varepsilon)}=Q / \varepsilon$. It follows from Dynkin formula that

$$
Y_{t}^{(\varepsilon)}=Y_{0}^{(\varepsilon)}+\frac{1}{\varepsilon} \int_{0}^{t} Y_{s}^{(\varepsilon)} Q d s+M_{t}
$$

where $\left(M_{t}\right)_{t \geq 0}$ is a $\mathcal{F}^{X^{(\varepsilon)}}$-martingale. Then applying $\left(\mathrm{Ch} \mathrm{IV}, \mathrm{Th} 1^{3}\right)$ to (12), we get for $\widehat{Y}_{t}^{(\varepsilon)}$

$$
\widehat{Y}_{t}^{(\varepsilon)}=\alpha+\frac{1}{\varepsilon} \int_{0}^{t} \widehat{Y}_{s}^{(\varepsilon)} Q d s+\widehat{M}_{t}
$$

where $\widehat{M}=\left(\widehat{M}_{t}\right)_{t \geq 0}$ is a $\mathcal{F}^{N^{(\varepsilon)}}$-martingale. Now, (Ch III, Th $\left.17^{3}\right)$ gives us the following representation of the $\mathcal{F}^{N^{(\varepsilon)}}$-martingale $\widehat{M}$

$$
\int_{0}^{t} G_{s}\left(d N_{s}^{(\varepsilon)}-\widehat{\lambda}_{s} d s\right)
$$

where $\left(G_{s}\right)_{s \geq 0}$ is a $\mathcal{F}^{N^{(\varepsilon)}}$-predictable process, called the innovations process. It remains to determine an explicit expression of $G_{s}$. This is similar to the case of a Poisson process governed by a Markov process (see pages 
$98,107^{3}$ ) else but $X^{(\varepsilon)}$ and $N^{(\varepsilon)}$ may have common jumps. So, we omit the details of the derivation. We have $G_{s}=G_{1, s}-G_{2, s}+G_{3, s}$ with

$$
\begin{aligned}
G_{2, s} & =\widehat{Y}_{s-}^{(\varepsilon)} \text { and } G_{1, s}=\left(\frac{\widehat{Y}_{s-}^{(\varepsilon)}(i)\left(D_{1} \mathbb{I}^{\mathrm{t}}\right)(i)}{\widehat{\lambda}_{s}}\right)_{i \in \mathcal{M}} \\
\text { and } G_{3, s} & =\left(\frac{\sum_{k \neq i} \widehat{Y}_{s-}^{(\varepsilon)}(k) D_{1}(k, i)-\widehat{Y}_{s-}^{(\varepsilon)}(i) \sum_{k \neq i} D_{1}(i, k)}{\widehat{\lambda}_{s}}\right)_{i \in \mathcal{M}}
\end{aligned}
$$

and the gain $G_{s}$ is of the form reported in Lemma 3 .

Equation (11) has the unique solution

$$
\widehat{Y}_{t}^{(\varepsilon)}=\alpha \exp (Q t / \varepsilon)+\int_{0}^{t} v_{s-}^{(\varepsilon)} \exp (Q(t-s) / \varepsilon)\left(d N_{s}^{(\varepsilon)}-\widehat{\lambda}_{s} d s\right) ;
$$

(check, with an integration by parts, that the right hand side term of (13) is a solution of (11) and verify that the difference of the two terms in (13) is a solution of a (first order) homogeneous linear differential equation with initial condition 0 ).

Theorem 4: Let $\left(P_{t}\right)_{t \geq 0}$ be the counting process of an HPP with the intensity $\left\langle\pi, D_{1} \mathbb{I}^{\mathrm{t}}\right\rangle$, where $\pi$ is the probability distribution such that $\pi Q=$ 0 . For any $T>0$, there exists a constant $C_{T}$ such that

$$
\mathrm{d}_{\mathrm{TV}}\left(\mathcal{L}\left(N_{\mathbb{T}}^{(\varepsilon)}\right), \mathcal{L}\left(P_{\mathbb{T}}\right)\right) \leq C_{T} \varepsilon
$$

Proof: Since $v_{s-}^{(\varepsilon)} \mathbb{I}^{\mathrm{t}}=0$, we can write from (13)

$$
\begin{aligned}
\left\|\widehat{Y}_{t}^{(\varepsilon)}-\pi\right\|_{1} \leq & \|\alpha \exp (Q t / \varepsilon)-\pi\|_{1} \\
& +\left\|\int_{0}^{t} v_{s-}^{(\varepsilon)}\left[\exp (Q(t-s) / \varepsilon)-\mathbb{I}^{t} \pi\right]\left(d N_{s}^{(\varepsilon)}-\widehat{\lambda}_{s} d s\right)\right\|_{1} .
\end{aligned}
$$

Since $Q$ is irreducible, we have the well known exponential estimate:

$$
t \geq 0, \quad\left\|\exp (Q t)-\mathbb{I}^{t} \pi\right\|_{1} \leq C \exp (-\rho t)
$$

where $\rho$ is positive and only depends on matrix $Q$.

In a first step, it follows from (14)

$$
\int_{0}^{T}\|\alpha \exp (Q t / \varepsilon)-\pi\|_{1} d t \leq C_{1} \varepsilon .
$$


In a second step, we have

$$
\begin{aligned}
& E\left\|\int_{0}^{t} v_{s-}^{(\varepsilon)}\left[\exp (Q(t-s) / \varepsilon)-\mathbb{I}^{\mathrm{t}} \pi\right]\left(d N_{s}^{(\varepsilon)}-\widehat{\lambda}_{s} d s\right)\right\|_{1} \\
\leq & 2 E \int_{0}^{t}\left\|v_{s-}^{(\varepsilon)}\left[\exp (Q(t-s) / \varepsilon)-\mathbb{I}^{\mathrm{t}} \pi\right]\right\|_{1} \widehat{\lambda}_{s} d s
\end{aligned}
$$

since $\widehat{\lambda}_{s}$ is the $\mathcal{F}^{N^{(\varepsilon)}}$-intensity of $N^{(\varepsilon)}$ and $v_{s-}^{(\varepsilon)}$ is $\mathcal{F}^{N^{(\varepsilon)}}$-predictable. Note that $\left\|v_{s-}^{(\varepsilon)}\right\|_{1} \widehat{\lambda}_{s}$ is uniformly bounded in $\varepsilon, t$. Moreover, using the exponential estimate (14) for $\left\|\exp (Q(t-s) / \varepsilon)-\mathbb{I}^{t} \pi\right\|_{1}$, we derive that, for all $t \geq 0$,

$$
E \int_{0}^{t}\left\|v_{s-}^{(\varepsilon)}\left[\exp (Q(t-s) / \varepsilon)-\mathbb{I}^{\mathrm{t}} \pi\right]\right\|_{1} \widehat{\lambda}_{s} d s \leq C_{2} t \varepsilon .
$$

We deduce from the previous estimate (and Fubini's theorem) that

$$
E \int_{0}^{T} \| \int_{0}^{t} v_{s-}^{(\varepsilon)}\left[\exp \left(Q(t-s) / \varepsilon-\mathbb{I}^{\mathrm{t}} \pi\right]\left(d N_{s}^{(\varepsilon)}-\widehat{\lambda}_{s} d s\right) \|_{1} d t \leq C_{2, T} \varepsilon .\right.
$$

Remark 5: With respect to Theorem 1 , note that Th $1^{8}$ would give the convergence in distribution of the counting process $N^{(\varepsilon)}$ to that of a Poisson process in the space of all counting processes, equipped with the Skorokhod topology. Moreover, convergence in variation also takes place in this space. Indeed, the distance in total variation over interval $[0, T]$ between the distributions of $N^{(\varepsilon)}$ and $P=\left(P_{t}\right)_{t \geq 0}$ is also bounded from above by $E \operatorname{Var}_{[0, T]}\left(\widehat{A}^{(\varepsilon)}-A\right)\left(\operatorname{Th} 4.1^{9}\right)$. Thus, it follows from Theorem 4 that the rate of convergence is in $\varepsilon$.

Remark 6: The order of the convergence rate in Theorem 4 cannot be improved in general. This follows from (Section 5, Example 1) ${ }^{5}$, where the authors report a lower bound for the distance in variation, that has order 1 in $\varepsilon$ for a Poisson process modulated by a 2-states Markov process. We refer the reader to ${ }^{5}$ for details.

\section{Conclusion}

In this chapter, we report some asymptotic properties of the well known Littlewood reliability model for modular softwares. Firstly, we deal with the stationary version of the failure point process, which is a classic concept in point process theory. Secondly, we prove a Poisson approximation for the point process when reliability growth takes place. This fact is reported in 14 but without proof ( $\mathrm{see}^{15}$ in the semi-Markov context). We also provide 
a convergence rate for the convergence of the point process to its Poisson limit.

All these results may be extended to a more general setting. For instance, Theorems 1 and 4 have their counterpart ${ }^{6}$ for a general architecturebased reliability model proposed by Ledoux. ${ }^{11}$ The purpose of this work was to take into account the way the failures may affect the execution process. We can also consider that failures can occur in batch. In such a case, using the martingale approach for marked point process, we get different approximating processes depending on the assumptions made on the batch size distributions. In the usual case of independent and identically distributed variables, we obtain a Compound Poisson process. This supports the use of software reliability models as considered by Sahninoglu ${ }^{21}$, when clumping of failures exists. Rate of convergence may also be provided. Details will be reported elsewhere.

\section{Acknowledgments}

The author would like to thank J.-B. Gravereaux for helpful discussions.

\section{References}

1. V. V. Anisimov and V.V. Korolyuk, Asymptotic reliability analysis of semiMarkov systems, Cybernetics, 18, 559-566 (1983).

2. S. Asmussen, Matrix-analytic models and their analysis, Scandinavian Journal of Statistics, 27, 193-226 (2000).

3. P. Bremaud, Point Processes and Queues (Springer, 1981).

4. E. Çinlar, Markov renewal theory, Advances in Applied Probability, 1, 123187 (1969).

5. G. B. Di Masi and Y. M. Kabanov, The strong convergence of two-scale stochastic systems and singular perturbations of filtering equations, Journal of Mathematical Systems, Estimation, and Control, 3, 207-224 (1993).

6. J.-B. Gravereaux and J. Ledoux, Poisson approximation for some reliability models, submitted (2002).

7. K. Goseva-Popstojanova and K. S. Trivedi, Architecture-based approach to reliability assessment of software systems, Performance Evaluation, 45, 179-204 (2001).

8. Y. M. Kabanov, R. S. Liptser and A. N. Shiryayev, Some limit theorems for simple point processes (martingale approach), Stochastics, 3, 203-216 (1980).

9. Y. M. Kabanov, R. S. Liptser and A. N. Shiryayev, Weak and strong convergence of the distributions of counting processes, Theory of Probability and its Applications, 28, 303-336 (1983).

10. J. Ledoux, Software reliability modeling, in Handbook of Reliability Engineering, Ed. H. Phoam (Springer, 2003) to appear. 
11. J. Ledoux, Availability modeling of modular software, IEEE Transactions on Reliability, 29, 159-168 (1999).

12. J. Ledoux and G. Rubino, Simple formulae for counting processes in reliability models, Advances in Applied Probability, 29, 1018-1038 (1997).

13. N. Limnios and G. Oprişan, Semi-Markov processes and Reliability (Series in Statistics for Industry and Technology, Birkhuser, 2001).

14. B. Littlewood, A reliability model for systems with Markov structure, $A p$ plied Statistics, 24, 172-177 (1975).

15. B. Littlewood, Software reliability model for modular program structure, IEEE Transactions on Reliability, 28, 241-246 (1979).

16. B. Littlewood, A software reliability model for modular program structure (supplement), ASIS-NAPS document No 03307 (1979).

17. D. M. Lucantoni, K. Meier and M. F. Neuts, A single-server queue with server vacations and a class of non-renewal arrival processes, Advances in Applied Probability, 22, 676-705 (1990).

18. S. Narayana and M. F. Neuts, The first two moment matrices of the counts for the markovian arrival process, Stochastic Models, 8, 459-477 (1992).

19. M. F. Neuts, Structured Stochastic Matrices of $M / G / 1$ Type and Their Applications (Marcel Dekker Inc., New-York and Basel, 1989).

20. R. Pyke, Markov renewal processes with finitely many states, Annals of Mathematical Statistics, 32, 1243-1259 (1961).

21. M. Sahninoglu, Compound-Poisson software reliability model, IEEE Transactions on Software Engineering, 18, 624-630 (1992).

22. N. D. Singpurwalla and S. P. Wilson, Statistical Methods in Software Engineering: Reliability and Risk (Springer, 1999). 